\title{
IUFOST2006/655 \\ Recent Advances on Modeling Turbulent Flow over Highly Perme- able Media: Applications to Food Industry
}

\author{
M. De Lemos \\ Instituto Tecnologico de Aeronautica - ITA, Departamento de Energia - IEME, 12228-900 Sao Jose dos \\ Campos, Brazil \\ delemos@ita.br
}

Food processing, transportation and marketing often require suitable refrigeration systems for adequate storage and conservation. Physical modeling of such storage stacks consider a permeable medium (food) through which a working fluid permeates (cooling air). As cooling requirements increase, an increase in the air mass low rate is necessary. Consequently, the air flow may become turbulent leading to difficulties in mathematically modeling the entire cooling process. This lecture shall review recent mathematical models for treating turbulent flow in permeable media. In particular, flow over a finite porous medium is investigated using different interfacial conditions. In such configuration, a macroscopic interface is identified between the two media. In the first model, no diffusion-flux is considered when treating the statistical energy balance at the interface. The second approach assumes that diffusion fluxes of turbulent kinetic energy on both sides of the interface are unequal. Comparing these two models, this paper presents numerical solutions for such hybrid medium, considering here a channel partially filled with a porous layer through which fluid flows in turbulent regime. One unique set of transport equations is applied to both regions. Effects of Reynolds number, porosity, permeability and jump coefficient on mean and turbulence fields are investigated. Results indicate that depending on the value of the stress jump parameter, substantially dissimilar fields for the turbulence energy are obtained. Negative values for the stress jump parameter give results closer to experimental data for the turbulent kinetic energy at the interface. 